\title{
Pressure-Induced Hydrogen-Hydrogen Interaction in Metallic FeH Revealed by NMR
}

\author{
Thomas Meier, ${ }^{1,{ }^{*}}$ Florian Trybel, ${ }^{1}$ Saiana Khandarkhaeva, ${ }^{1}$ Gerd Steinle-Neumann, ${ }^{1}$ Stella Chariton, ${ }^{1}$ Timofey Fedotenko, ${ }^{2}$ \\ Sylvain Petitgirard, ${ }^{3}$ Michael Hanfland, ${ }^{4}$ Konstantin Glazyrin, ${ }^{5}$ Natalia Dubrovinskaia, ${ }^{2}$ and Leonid Dubrovinsky ${ }^{1}$ \\ ${ }^{1}$ Bayerisches Geoinstitut, University of Bayreuth, D-95447 Bayreuth, Germany \\ ${ }^{2}$ Material Physics and Technology at Extreme Conditions, Laboratory of Crystallography, \\ University of Bayreuth, D-95447 Bayreuth, Germany \\ ${ }^{3}$ Institute of Geochemistry and Petrology, Department of Earth Sciences, \\ Eidgenössische Technische Hochschule Zürich, S-8092, Switzerland \\ ${ }^{4}$ European Synchrotron Radiation Facility (ESRF), F-38043 Grenoble Cedex, France \\ ${ }^{5}$ Deutsches Elektronen-Synchrotron (DESY), D-22603, Hamburg, Germany
}

(Received 4 March 2019; revised manuscript received 13 May 2019; published 17 July 2019)

\begin{abstract}
Knowledge of the behavior of hydrogen in metal hydrides is the key for understanding their electronic properties. Here, we present an ${ }^{1} \mathrm{H}-\mathrm{NMR}$ study of cubic FeH up to $202 \mathrm{GPa}$. We observe a distinct deviation from the ideal metallic behavior between 64 and $110 \mathrm{GPa}$ that suggests pressure-induced $\mathrm{H}-\mathrm{H}$ interactions. Accompanying $a b$ initio calculations support this result, as they reveal the formation of an intercalating sublattice of electron density, which enhances the hydrogen contribution to the electronic density of states at the Fermi level. This study shows that pressure-induced $\mathrm{H}-\mathrm{H}$ interactions can occur in metal hydrides at much lower compression and larger $\mathrm{H}-\mathrm{H}$ distances than previously thought and stimulates an alternative pathway in the search for novel high-temperature superconductors.
\end{abstract}

DOI: 10.1103/PhysRevX.9.031008

\section{INTRODUCTION}

Hydrides and hydrogen-rich compounds continue to attract considerable attention as the search for effective and "green energy" materials intensifies [1,2]. Recent theoretical [3], computational [4-6], and experimental $[7,8]$ results indicate that hydrides may hold the key to a deep understanding of high-temperature superconductivity and the synthesis of compounds exhibiting high critical temperatures $\left(T_{c}\right)$. Sulphur hydride $\left(\mathrm{H}_{3} \mathrm{~S}\right)$, with a $T_{c}$ of $200 \mathrm{~K}$ at a pressure $(P)$ of approximately $150 \mathrm{GPa}$ [9], is a prominent example of such metal-hydride (MH) systems.

A systematic analysis of computational results $[10,11]$ suggests that two properties are particularly important for achieving high- $T_{c}$ superconductivity in metallic $\mathrm{MH}$ : (i) a significant contribution of hydrogen to the electronic density of states at the Fermi energy $N\left(E_{F}\right)$ and (ii) strong effects of hydrogen vibrations on the electronic structure of the material (i.e., electron-phonon coupling). Unfortunately, the lack of experimental methods that provide access to electronic states of hydrogen in MHs at such $P$ prohibits a direct confirmation of this hypothesis.

*thomas.meier@uni-bayreuth.de

Published by the American Physical Society under the terms of the Creative Commons Attribution 4.0 International license. Further distribution of this work must maintain attribution to the author(s) and the published article's title, journal citation, and DOI.
Subject Areas: Condensed Matter Physics

Recent developments in our group led to the implementation of nuclear magnetic resonance (NMR) spectroscopy in diamond anvil cells (DACs) at pressures approaching $100 \mathrm{GPa}$ using magnetic flux tailoring Lenz lenses [12-14]. NMR spectroscopy is widely recognized [15-19] for its sensitivity to small electronic effects. In particular, Knight shift measurements provide a well-established technique to investigate the density of states of conduction electrons at the Fermi energy, enabling the detection of deviations from free-electron gas behavior or even electronic topological transitions of the Fermi surface and direct evidence of the MeissnerOchsenfeld effect in the metal's superconducting state.

In density-functional-theory-based calculations, Peng et al. [20] demonstrate that in clathratelike metal hydrogen systems, such as $\mathrm{LaH}_{10}$ or $\mathrm{YH}_{10}$, the nearest H-H distances should be $\leq 1.5 \AA$ for the development of significant hydrogen-hydrogen interactions. In nonmagnetic fcc $\mathrm{FeH}$, stable above $25 \mathrm{GPa}$ [21], average $\mathrm{H}-\mathrm{H}$ distances are in the range of 2.6-2.3 $\AA$ at $200 \mathrm{GPa}$ [22], suggesting that $P$-induced $\mathrm{H}-\mathrm{H}$ correlations are unlikely. As the synthesis of fcc FeH in a DAC is well established and the crystal structure well defined, hydrogenation and composition of $\mathrm{FeH}$ samples can be closely controlled. Therefore, fcc iron monohydride can be regarded as a reference system for our NMR-based approach to the investigation of the electronic structure of hydrogen in metal hydrides. 


\section{EXPERIMENTAL METHODS}

Two identical DACs of type BX90 [23] are prepared with two beveled diamond anvils with $100 \mu \mathrm{m}$ culets. Rhenium gaskets are indented to about $15 \mu \mathrm{m}$ thickness, and $40 \mu \mathrm{m}$ holes are laser cut in the flat face of the preindentation to form the sample cavity. Using physical and chemical vapor deposition techniques, the anvils are covered by a $1 \mu \mathrm{m}-$ thick layer of copper, and the metallic rhenium gaskets are sputtered with a $500 \mathrm{~nm}$-thick layer of $\mathrm{Al}_{2} \mathrm{O}_{3}$ for electrical insulation. Subsequently, using a focused ion beam, three Lenz lenses (LLs) are cut out of the copper layers covering the diamond anvils: (i) along the anvil's pavilion, (ii) on the beveled area, and (iii) on the $100 \mu \mathrm{m}$ culets [Fig. 1(a)]. This procedure is necessary to avoid damage due to sharp edges from the $8^{\circ}$ bevel and the culet rim when cupping under compression occurs. The nuclear magnetic resonators used in this study can be regarded as a generalization of the recently introduced double-stage Lenz lenses [14], enabling its use for single-beveled diamond anvils with culet sizes of $\leq 100 \mu \mathrm{m}$.

The cells are loaded by flooding the sample chamber with paraffin oil and then mixing fine iron powder of natural composition (Sigma-Aldrich, 4N purity) into it. Pressure in the sample chamber is estimated by the frequency shift of the first-order Raman peak of the diamond at its edge in the center of the sample cavity [Fig. 1(b)] [24,25].

The radio-frequency (rf) excitation coils are made using two identical $3 \mathrm{~mm}$ coils consisting of five turns of $150 \mu \mathrm{m}$
Teflon-insulated copper wire. Each coil is fixed around the diamonds on the respective backing plates and connected to form a Helmholtz coil, ensuring optimal inductive coupling into the LL resonator structures. NMR measurements are conducted at a magnetic field of $1 \mathrm{~T}$ using a sweepable electron spin resonance magnet with a 50-mm magnet pole distance and a Techmac Redstone spectrometer for solidstate NMR applications. NMR measurements prior to cell loading do not exhibit any detectable hydrogen signals within typical experimental parameters, showing the absence of spurious hydrogen signals stemming from other parts of the NMR probe.

From rf nutation experiments, an optimal $90^{\circ}$ pulse of $4 \mu \mathrm{s}$ at an average pulse power of $40 \mathrm{~W}$ is established. This value does not change over the course of the experiment and is found to be valid for both DACs used. For measurements of spin-lattice relaxation rates, a saturation recovery pulse train consisting of 16 consecutive pulses is used. From the relation $B_{1}=\pi /\left(2 \gamma_{n} t_{\pi / 2}\right)$, with $\gamma_{n}$ the nuclear gyromagnetic ratio and $t_{\pi / 2}$ the $\pi / 2$ pulse length, a $B_{1}$ field of $4.7 \mathrm{mT}$ is deduced. Using the FEMM 4.2 package, the high-frequency $B_{1}$ field distribution across the $40 \mu \mathrm{m} \times 15 \mu \mathrm{m}$ sample cavity is calculated using experimental parameters similar to the nutation experiments. An average $B_{1}$ field of $4.8 \mathrm{mT}$ is found, in excellent agreement with the nutation experiments. The introduced LL resonators show the required sensitivity.

All resonance frequency shifts are referenced relative to the hydrogen signal of the paraffin hydrogen reservoir

(a)
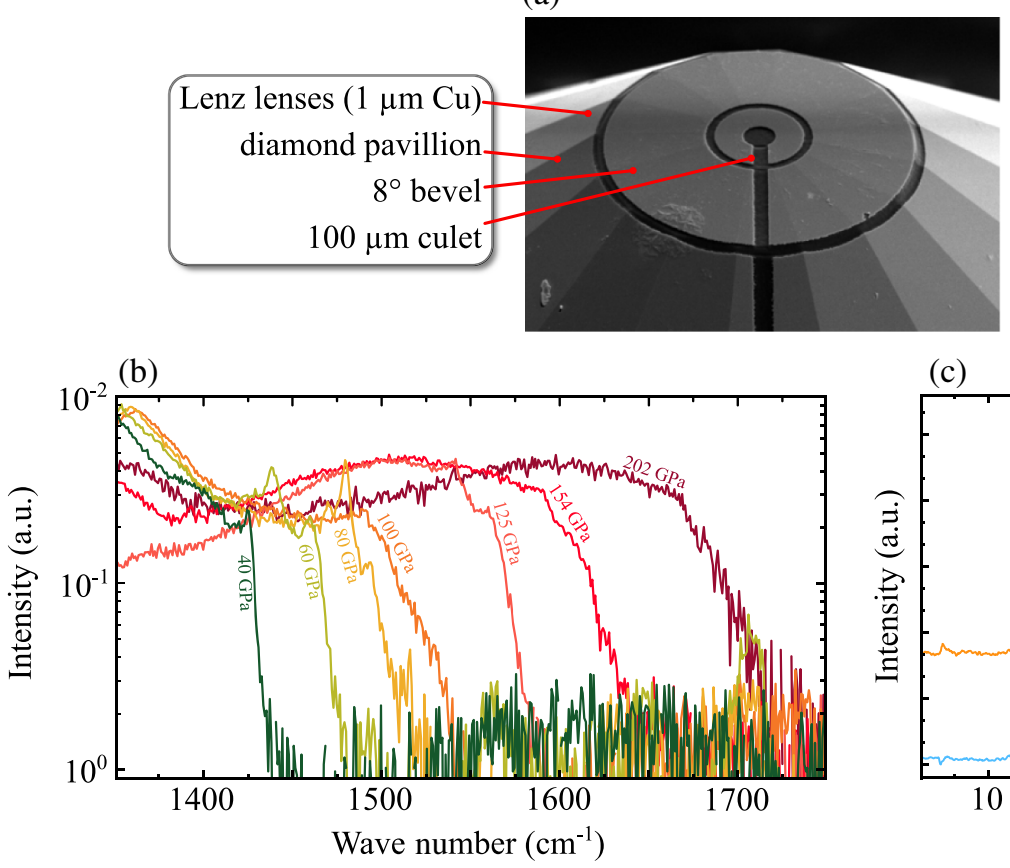

(c)

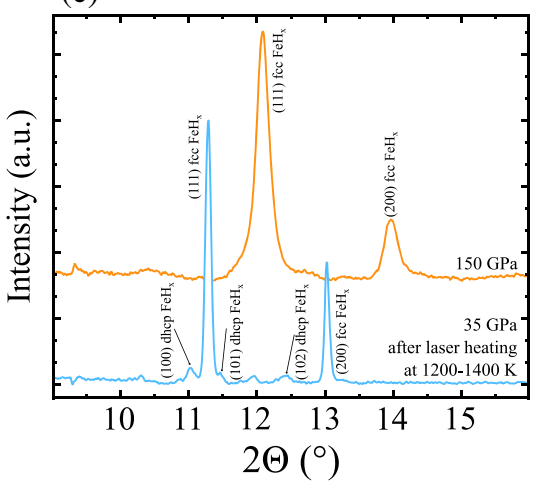

FIG. 1. (a) SEM image of the NMR Lenz lens resonator structure deposited and formed on the surface of a 100- $\mu \mathrm{m}$ diamond anvil, (b) Raman spectra of the diamond edge at the center of the 100- $\mu$ m culets used for pressure calibration, and (c) $\mathrm{x}$-ray diffraction patterns after sample synthesis (laser heating) at $35 \mathrm{GPa}$ (blue line) and after compression to $150 \mathrm{GPa}$ (orange line). 


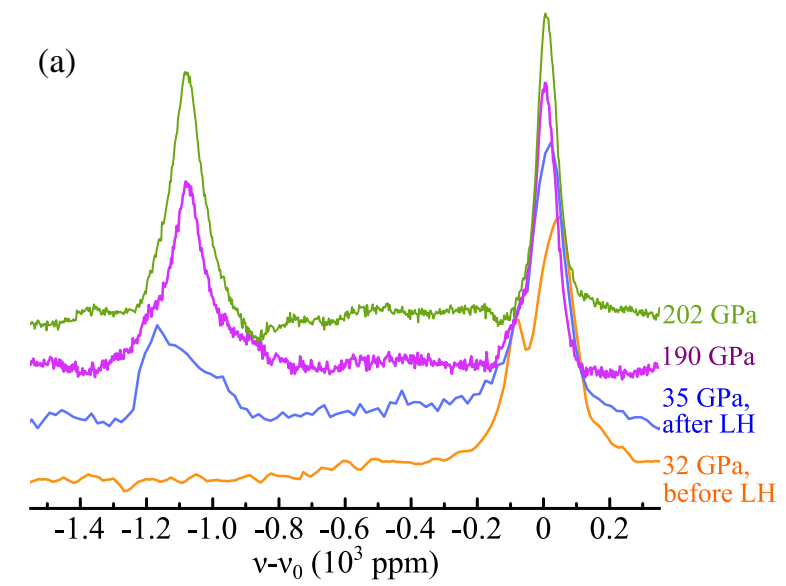

(b)

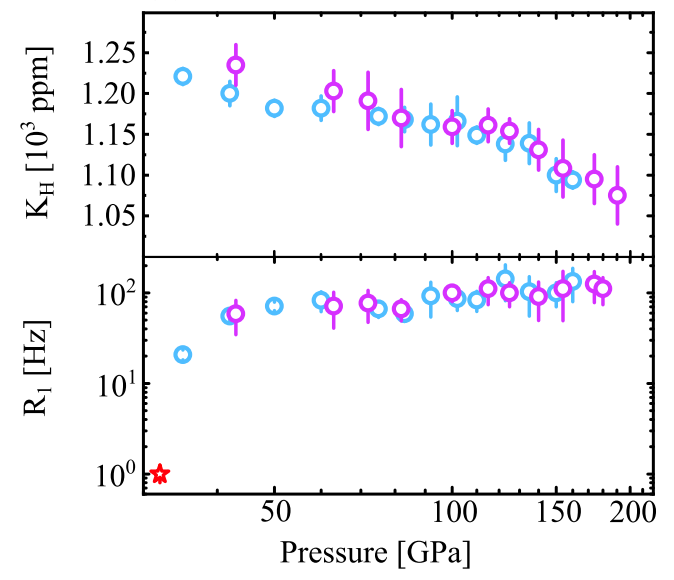

(c)

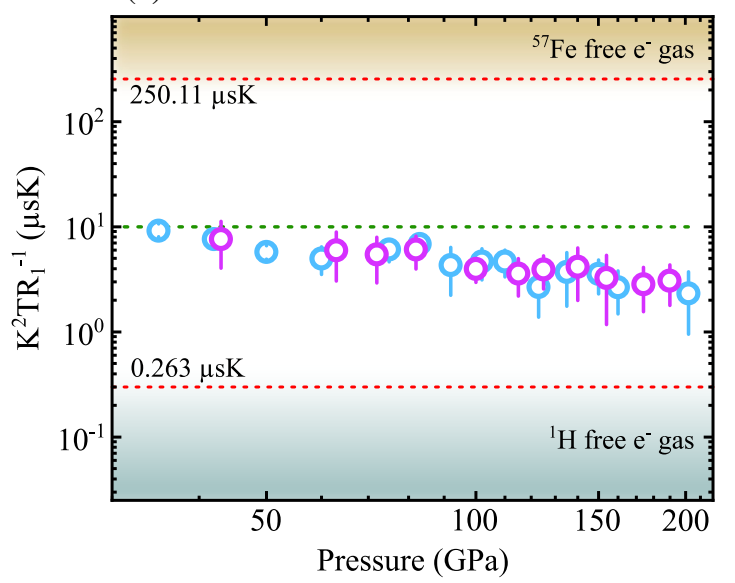

FIG. 2. (a) Hydrogen NMR spectra. Shown are spectra before laser heating at $32 \mathrm{GPa}$ (orange line), directly after laser heating (blue line), and after compression to 190 and $202 \mathrm{GPa}$ (purple and green line, respectively). (b) Summary of Knight shift $K_{H}$ relative to the hydrogen reservoir (top) and the spin-lattice relaxation rates $R_{1}$ (bottom). Blue and purple data points refer to data collected in cells 1 and 2, respectively. The red data point refers to the relaxation rate of the hydrogen reservoir prior to sample synthesis. (c) Pressure dependence of the Korringa ratio. Shaded areas qualitatively depict the regions expected for pure iron (top) and metallic hydrogen (bottom).

after laser heating. The values for the Knight shift (Fig. 2) are higher by a factor of 6 than for other transition metal hydrides [26]. The choice of the reference material can, in principle, lead to an intrinsic overestimate as we deviate from the standard shift reference, tetramethylsilane (TMS) [27]. However, TMS does not induce the reaction to form $\mathrm{FeH}$ at a high $P$ and temperature ( $T$ ), to the best of our knowledge.

The DACs are pressurized initially to about $30 \mathrm{GPa}$, into the stability field of nonmagnetic hcp Fe, and subsequently laser heated to $1200 \mathrm{~K}$. X-ray diffraction (XRD) powder patterns taken at PETRA III (beam line P02) and ESRF (ID-15) show reflections from $\mathrm{FeH}$ and no residual $\mathrm{Fe}$ signal [Fig. 1(c)], suggesting that iron has fully reacted with hydrogen from the paraffin reservoir. The most prominent diffraction peaks are those of fcc $\mathrm{FeH}$ previously identified $[21,28]$; a small amount of dhcp $\mathrm{FeH}_{x}$ is also indexed.

As fcc $\mathrm{FeH}_{x}$ is unstable at ambient conditions, the actual chemical composition cannot be measured directly using recovered samples. Narygina et al. [21] suggest that the hydrogenation factor $x$ can be estimated by considering the volume expansion of the fcc unit cell in response to the dissolution of hydrogen on the octahedral interstitial lattice sites of fcc Fe by

$$
x=\left(V_{\mathrm{FeH}_{x}}-V_{\mathrm{Fe}}\right) / \Delta V_{\mathrm{H}},
$$

with $\mathrm{V}_{\mathrm{FeH}_{x}}$ and $V_{\mathrm{Fe}}$ the atomic volumes of iron hydride and fcc iron, respectively. $\Delta V_{\mathrm{H}}$ is the volume expansion due to hydrogenation. As the latter parameter is not well defined by other fcc-structured $3 d$ transition metals at ambient conditions, the actual hydrogenation value $x$ is estimated by comparing the recorded data with equations of state of $\mathrm{FeH}$ [21] and fcc iron [29]. Using this procedure, $x=1.0(1)$ in both DACs is found, close to stoichiometry. Further XRD measurements at $150 \mathrm{GPa}$ suggest that fcc $\mathrm{FeH}$ remains stable with similar composition throughout the whole $P$ range of this study.

Prior to laser heating, ${ }^{1} \mathrm{H}-\mathrm{NMR}$ spectra are recorded [Fig. 2(a)]: As the origin of the hydrogen signal is the 
$\mathrm{C}_{n} \mathrm{H}_{2 n+2}$ reservoir, a single NMR signal positioned at the Larmor frequency for hydrogens is observed. The center of gravity of the paraffin signal is found at $45.0737 \mathrm{MHz}$, corresponding to a polarizing magnetic field of $B_{0}=$ $1.058 \mathrm{mT}$ within the sample cavity. Small deviations of up to $10 \%$ in $B_{0}$ are found to originate from a slightly different positioning of the DACs within the region of the highest magnetic field of the electromagnet but can be neglected, since the hydrogen reservoir serves as an internal reference. The recorded spectra of paraffin at 32 (cell 1) and $29 \mathrm{GPa}$ (cell 2) are found to be similar to earlier ${ }^{1} \mathrm{H}-\mathrm{NMR}$ spectra of $\mathrm{C}_{n} \mathrm{H}_{2 n+2}$ in terms of linewidths and relaxation rates [12]. After laser heating, an additional intense signal is found at lower frequencies at about $-1200 \mathrm{ppm}$ relative to the hydrogen reference [Fig. 2(a)]. Comparing signal intensities from spectra recorded after full relaxation of both independent spin systems, we determine an intensity ratio of about $4: 7$ of $\mathrm{FeH}$ to $\mathrm{C}_{n} \mathrm{H}_{2 n+2}$ in cell 1 and $1: 5$ in cell 2. These ratios are found to be constant at all $P$, suggesting that further reaction of hydrogen with the iron powder during cold compression cycles does not occur.

\section{COMPUTATIONAL DETAILS}

All $a b$ initio calculations performed are based on the Kohn-Sham density functional theory and are performed with QUANTUM ESPRESSO [30,31] using the projector augmented wave approach [32]. We use the generalized gradient approximation by Perdew-Burke-Ernzerhof [33] to exchange and correlation with corresponding potential files; for Fe, we use a valence electron configuration that includes electronic states $3 s$ and higher, applicable to extreme pressure [34].

Convergence tests for the electronic band structure and density of state calculations lead to reciprocal space sampling with a Monkhorst-Pack [35] $k$-point grid of $32 \times$ $32 \times 32$ and a cutoff energy for the plane wave expansion of $120 \mathrm{Ry}$. In addition to the total electronic density of states, site and orbital projections are computed, with particular attention to the crystal field splitting of the $3 d$ electronic states into the $e_{g}$ and $t_{2 g}$ orbitals.

In order to identify regions of charge localization, we use the electron localization function (ELF) [36] that quantifies the probability of finding two electrons in close proximity by the ratio of a computed charge density and its spatial derivative to the value of the homogeneous electron gas of the same density.

\section{KNIGHT SHIFT IN IRON HYDRIDE}

As both stable iron hydride phases, fcc and dhcp, are thought to be metallic [37,38], the influence of hyperfine interaction of conduction electrons with the nuclei must be taken into account to explain the NMR signal. For transition metal hydrides, the magnetic susceptibility $(\chi)$ can be written as a sum of several contributions, $\chi=\chi_{s}+\chi_{d}+\chi_{o}+\chi_{\text {dia }}$, stemming from spin interactions with $s$ - or $d$-conduction electrons, the orbital paramagnetic, and the diamagnetic contribution of the ion cores, respectively. Therefore, the electron-nuclear hyperfine interaction, described in terms of the Knight shift $K_{\text {tot }}$ and the spin-lattice relaxation rate $R_{1}$ $[39,40]$, are caused by various mechanisms [41]:

$$
\begin{aligned}
K_{\mathrm{tot}} & =K_{s}+K_{d}+K_{o} \\
& =\frac{1}{A \mu_{B}}\left(H_{\mathrm{hf}}^{s} \chi_{s}+H_{\mathrm{hf}}^{d} \chi_{d}+H_{\mathrm{hf}}^{o} \chi_{o}\right)
\end{aligned}
$$

and

$$
\begin{aligned}
R_{1}= & 4 \pi \mu_{0}^{2} \gamma_{n}^{2} \hbar k_{B} T\left\{\left[H_{\mathrm{hf}}^{s} N_{s}\left(E_{F}\right)\right]^{2}\right. \\
& \left.+\left[H_{\mathrm{hf}}^{d} N_{d}\left(E_{F}\right)\right]^{2} q+\left[H_{\mathrm{hf}}^{o} N_{d}\left(E_{F}\right)\right]^{2} p\right\},
\end{aligned}
$$

where $A$ is Avogadro's constant and $\mu_{B}$ the Bohr magneton. The hyperfine fields $H_{\mathrm{hf}}^{s}, H_{\mathrm{hf}}^{d}$, and $H_{\mathrm{hf}}^{o}$ at the nucleus under investigation originate from the following mechanisms: (i) contact interaction of $s$ conduction electrons with the nucleus, (ii) core polarization of $s$ orbitals caused by uncompensated $d$ electrons, and (iii) the effect of orbital motions of $d$ electrons. $N_{s}\left(E_{F}\right)$ and $N_{d}\left(E_{F}\right)$ in Eq. (2) are the $s$ - and $d$-electron density of states at the Fermi energy, respectively. The factors $p$ and $q$ are reduction factors depending on $d$-orbital degeneracy at $E_{F}$.

For a free-electron Fermi gas, we can substitute $\chi_{(s, d)}=$ $2 \mu_{0} \mu_{B}^{2} N_{(s, d)}\left(E_{F}\right)$ in Eq. (2), leading to a reduced representation of the Knight shift:

$$
\begin{aligned}
K_{\mathrm{tot}}= & 2 \mu_{0} \mu_{B}\left[H_{\mathrm{hf}}^{s} N_{s}\left(E_{F}\right)+H_{\mathrm{hf}}^{d} N_{d}\left(E_{F}\right)\right] \\
& +\frac{1}{A \mu_{B}} H_{\mathrm{hf}}^{o} \chi_{o} .
\end{aligned}
$$

Equation (4) demonstrates that various mechanisms producing hyperfine fields at the hydrogen site need to be taken into account. However, as the $1 s$ electronic states of hydrogen and the $4 s$ states of iron typically lie far below and above the Fermi energy, respectively, it seems reasonable to assume their contribution to $K_{\text {tot }}$ to be negligible to first approximation in $\mathrm{FeH}$.

The effect of orbital motion of $3 d$ electrons on the hydrogen Knight shift $\left(K_{H}\right)$ is expected to be virtually nonexistent, as such an interaction would dominate Eq. (4) and lead to positive values of $K_{H}$ which are not observed in other $\mathrm{MH}$ systems measured at ambient conditions [41-44]. Also, considering the good correlation between $K_{H}$ and $N_{t_{2 g}}\left(E_{F}\right)$ (discussed in greater detail below), an influence of spin orbit coupling on the ${ }^{1} \mathrm{H}-\mathrm{NMR}$ spectra can be ruled out (Fig. 3). Therefore, both Eqs. (3) and (4) are reduced to their $d$-electron core polarization contribution, with a Korringa-type [45] relation given by

$$
\frac{K_{H}^{2} T}{R_{1}}=\frac{\hbar}{4 \pi k_{B}}\left(\frac{\gamma_{e}}{\gamma_{n}}\right)^{2} q .
$$




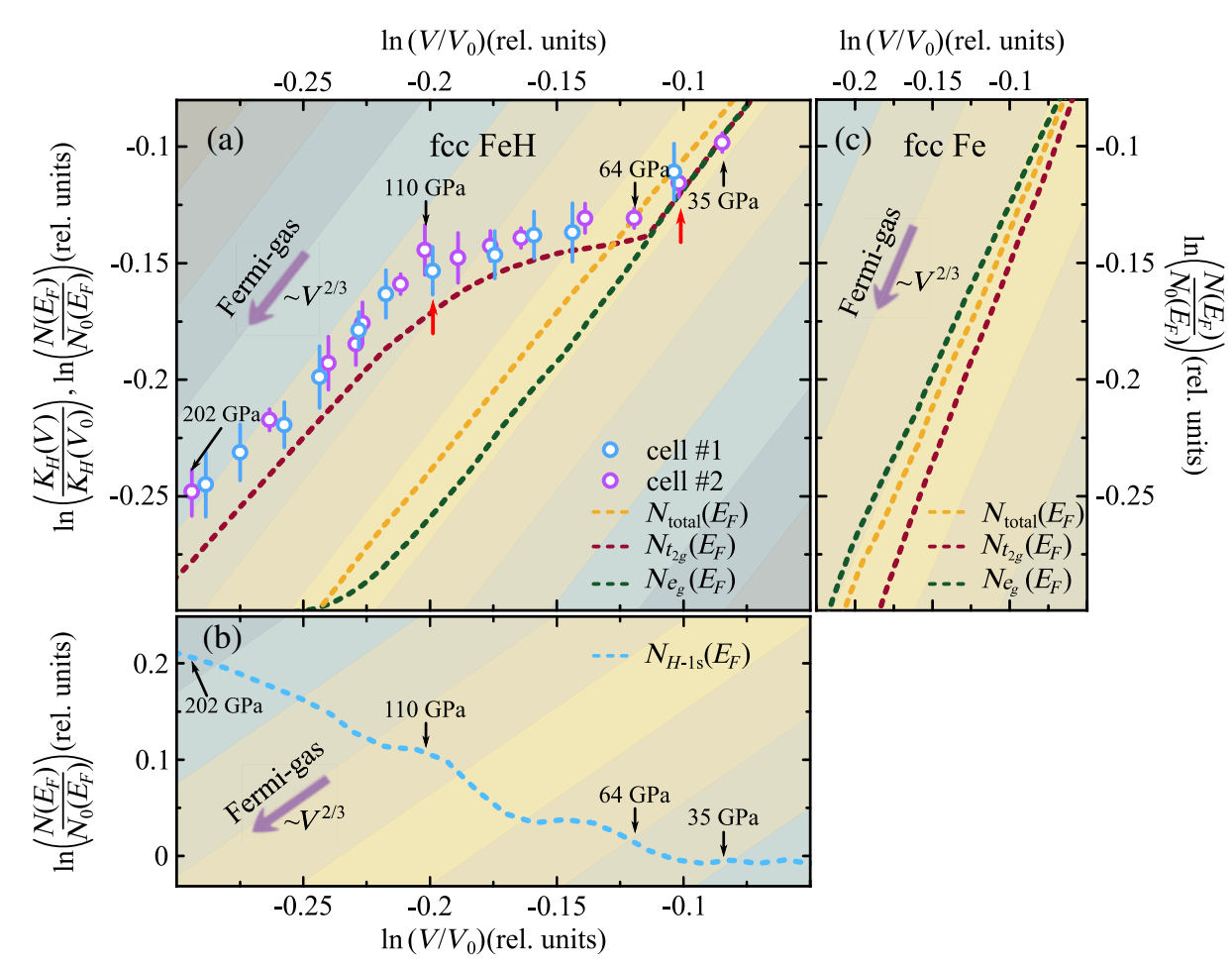

FIG. 3. Comparison of experimental data and ab initio electronic density of states calculations. (a) Double logarithmic power plot of relative changes in $K_{H}$ and $N\left(E_{F}\right)$ as a function of the relative volume. Experimental data points (blue and purple) have been normalized to $V_{0}$ using the equation of state from Narygina et al. [21] and extrapolated values of $K_{H}\left(V_{0}\right)$. Note that using complementary equation of state data from Thompson et al. [28] or Pépin et al. [22] does not affect our conclusions. The dashed lines (splines through computed values) show the dependence of $N_{\text {total }}\left(E_{F}\right), N_{t_{2 g}}\left(E_{F}\right)$, and $N_{e_{g}}\left(E_{F}\right)$. The diagonal color strips are guides to the eye depicting a $\propto V^{2 / 3}$ scaling for free-electron Fermi-gas-like behavior [as $N(E) \propto V \sqrt{E}$ and $E_{F} \propto V^{-2 / 3}$ [46], $N\left(E_{F}\right) \propto V \cdot \sqrt{V^{-2 / 3}} \propto V^{2 / 3}$ ]. Black arrows denote respective pressures points; red arrows show data recorded under decompression. (b) Evolution of $N_{H-1 s}\left(E_{F}\right)$. Indicated pressure markers are related to the experimental data points from (a). (c) Volume dependence of $N_{\text {total }}\left(E_{F}\right), N_{t_{2 g}}\left(E_{F}\right)$, and $N_{e_{g}}\left(E_{F}\right)$ for fcc Fe over a similar compression range.

For cubic lattices, the reduction factor $q$ can be described by $q=\frac{1}{3} f^{2}+\frac{1}{2}(1-f)^{2}$, where $f$ is the fractional character of $t_{2 g}$ orbitals at the Fermi surface [47,48], determining the deviation from the free-electron value given by $S=$ $\left[\hbar /\left(4 \pi k_{B}\right)\right]\left(\gamma_{e} / \gamma_{n}\right)^{2}[15]$, with $\gamma_{e}$ the gyromagnetic ratio of the electron.

In general, the Korringa-ratio constitutes an indicator for metallicity of a given material [15] using the volume independence of Eq. (5). As the Korringa ratio depends on only physical constants, values for the free-electron gas behavior for every metallic system can be readily estimated. For $\mathrm{FeH}$, two different limiting scenarios can be identified: (i) pure ${ }^{57} \mathrm{Fe}$ metal with $K^{2} T / R_{1}=250.11 \mu \mathrm{sK}$ and (ii) electronic Fermi-gas-like behavior of metallic hydrogen with a value of $0.263 \mu \mathrm{sK}$. The Korringa ratio for the binary system consisting of $\mathrm{Fe}$ and $\mathrm{H}$ can be anticipated to lie in between these extreme values.

\section{RESULTS AND DISCUSSION}

At approximately $30 \mathrm{GPa}$ and before laser heating, only a single NMR signal is observed [Fig. 2(a)], originating from the paraffin hydrogen reservoir which has been characterized in earlier experiments [12]. After $\mathrm{FeH}$ synthesis at $1200 \mathrm{~K}$, an additional ${ }^{1} \mathrm{H}-\mathrm{NMR}$ signal appears at about $-1200 \mathrm{ppm}$ relative to the paraffin hydrogen reservoir (Fig. 2). If this huge shift is due to the occurrence of $K_{H}$ in the metallic state [39], nuclear relaxation, as described by Heitler and Teller [49], must occur as well. We observe, indeed, that, in response to $\mathrm{FeH}$ synthesis, the spin-lattice relaxation rates $\left(R_{1}\right)$ increase by more than one order of magnitude from approximately $1 \mathrm{~Hz}$ in the hydrogen reservoir to approximately $20 \mathrm{~Hz}$ in the spin system associated with the additional signal [Fig. 2(b)].

Combining shift and relaxation rates, the Korringa ratio [Eq. (5)] should yield values intermediate to those for pure ${ }^{57} \mathrm{Fe}$ metal and a Fermi-gas-like value of the proton site [Fig. 3(c)]. The value of the Korringa ratio recorded directly after sample synthesis is found to be approximately $10 \mu \mathrm{sK}$, halfway in magnitude between these two values [Fig. 2(c)]. Therefore, the appearance of the additional signal can be attributed to the formation of metallic $\mathrm{FeH}$ in the DAC. Similar ${ }^{1} \mathrm{H}-\mathrm{NMR}$ spectra of other metal-hydride systems, synthesized at ambient conditions, confirm this 

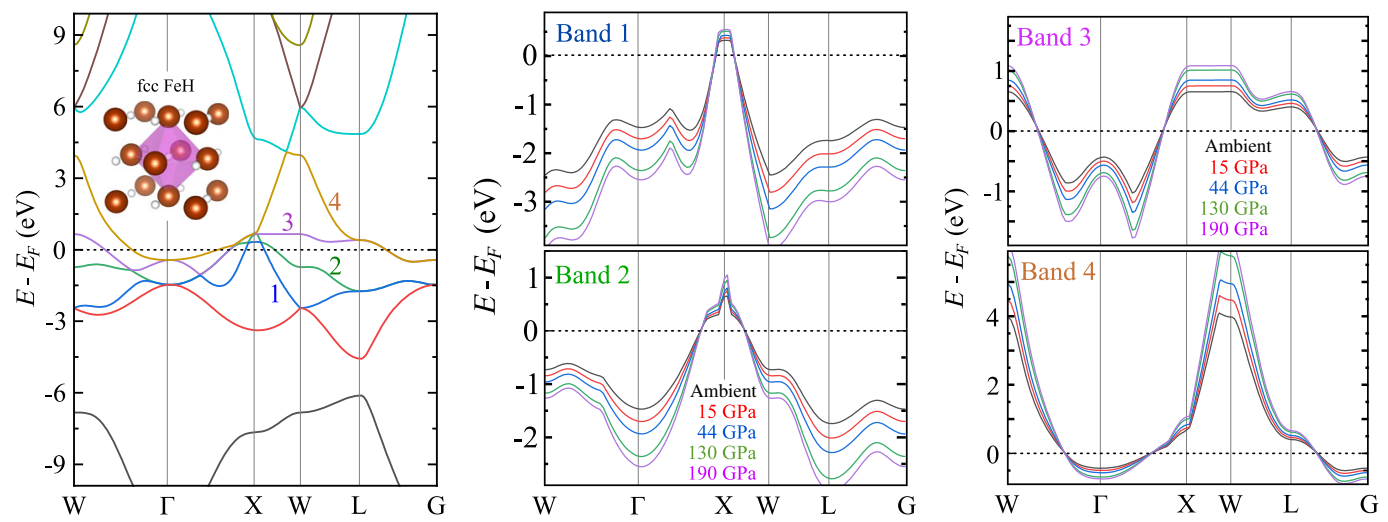

FIG. 4. Calculated band structure of fcc FeH. The left panel shows the band structure at ambient pressure, the two right panels the pressure evolution of four bands crossing the Fermi energy $\left(E_{F}\right)$, identified on the left.

conclusion [41,50]. Figure 2(c) also shows the pressure evolution of the Korringa ratio which should be volume independent in metallic systems [Eq. (5)]. However, a distinct $P$ dependence is observed between 30 and $202 \mathrm{GPa}$. To investigate this phenomenon, we focus on the analysis of the volume dependence of $K_{H}$.

Figure 3 shows a double logarithmic power plot of the relative volumes, using the equation of state from Narygina et al. [21], against relative changes in the Knight shift. Three distinct regions can be identified: (i) Up to $64 \mathrm{GPa}$, the exponent of the Knight shift ratio closely follows a Fermi-gas electronic behavior with $\propto V^{2 / 3}$, (ii) a deviation from metallic scaling exponents occurs between 64 and $110 \mathrm{GPa}$, and (iii) the reoccurrence of free-electron gas behavior from $110 \mathrm{GPa}$ to highest $P$. After compression and data collection up to $202 \mathrm{GPa}$, one of the DAC experiments is decompressed to approximately $100 \mathrm{GPa}$ and subsequently to $33 \mathrm{GPa}$ [red arrows in Fig. 3(a)]. NMR spectra collected on decompression agree with those obtained during compression, showing that the observed effect is fully reversible. As x-ray diffraction data do not show any sign of a structural phase transition [22,28], the origin of this observation should be linked to changes in the electronic environment of the hydrogens in $\mathrm{FeH}$.

The computed electronic band structure (Fig. 4) and density of states do not indicate any discontinuities close to $E_{F}$, such as van Hove singularities [51]. Therefore, topological transitions of the Fermi surface [52], which would influence both spin shift and relaxation rates, can be excluded. Values of the total density of states at $E_{F}$, $N_{\text {total }}\left(E_{F}\right)$, as well as its projection on the $3 d-t_{2 g}$ and $e_{g}$ states, $N_{t_{2 g}}\left(E_{F}\right)$ and $N_{e_{g}}\left(E_{F}\right)$, respectively, from the calculations are included in Fig. 3(a). The volume dependence of $N_{t_{2 g}}\left(E_{F}\right)$ closely follows the NMR spin shifts to $64 \mathrm{GPa}$. Above this $P$, experimental data points and $N_{t_{2 g}}\left(E_{F}\right)$ diverge beyond experimental errors.

The hydrogen Knight shift is the sum of interactions due to core polarization of uncompensated $d$ orbitals and contact interaction of $s$ electrons with the nuclei [Eq. (4)]. At ambient conditions, the latter interaction can be regarded as negligible due to the density of states at $E_{F}$ being almost zero for the hydrogen $1 s$ electron, $N_{H-1 s}\left(E_{F}\right)$ [Fig. 3(b)]. The contribution of $N_{H-1 s}\left(E_{F}\right)$ to the total density of states begins to increase around $64 \mathrm{GPa}$ and continuously rises with $P$, up to $202 \mathrm{GPa}$, the highest $P$ considered. This effect can qualitatively explain the observed offset between experimental data and calculated density of state values.

Figure 3(c) shows the respective volume dependence of the density of states at $E_{F}$ of the $3 d$ electrons for fcc-Fe over the same compression range as the $\mathrm{FeH}$ data. The $P$-dependent effects observed on the density of states of the $3 d-t_{2 g}$ orbitals in fcc FeH [Fig. 3(a)] are not present in fcc Fe. This difference allows us to conclude that they are caused by the presence of hydrogen atoms. Both the scaling anomaly in $N_{t_{2 g}}\left(E_{F}\right)$ and the continuous increase in $N_{H-1 s}\left(E_{F}\right)$ with compression suggest a shift of conduction electron density between iron and hydrogen atoms in $\mathrm{FeH}$.

In order to elucidate this possibility, we map the ELF in three different crystal planes [( $\left.\begin{array}{lll}1 & 0 & 0\end{array}\right),\left(\begin{array}{lll}1 & 1 & 0\end{array}\right)$, and $\left.\left(\begin{array}{lll}1 & 1 & 2\end{array}\right)\right]$ at compressions between $V / V_{0}=1.0$ and $V / V_{0}=0.61$ (21 steps) and consider the correlations between the evolution of the ELF maps and the data shown in Fig. 3 (Movie S1 in Supplemental Material [53]); Fig. 5 presents the ELF maps for $V / V_{0}=0.9$ and $V / V_{0}=0.69$, corresponding to approximately 30 and approximately $200 \mathrm{GPa}$, respectively.

At $V / V_{0}=0.9$, electron clouds associated with Fe and $\mathrm{H}$ atoms are localized, evidencing the ionic character of FeH. Regions exhibiting Fermi-gas electronic behavior, i.e., ELF values of 0.5, start to develop and spread at $V / V_{0}<0.88$, which are particularly pronounced in the (110) and (112) planes. These regions of increased electron density connect at $V / V_{0}<0.78$, corresponding to pressures of 80-90 GPa, forming an interconnecting network with respect to the hydrogen nuclei. At $V / V_{0}<0.73$, the nearest-neighbor $\mathrm{H}-\mathrm{H}$ bonds are bridged as well. Strikingly, the deviation from free-electron gas behavior 

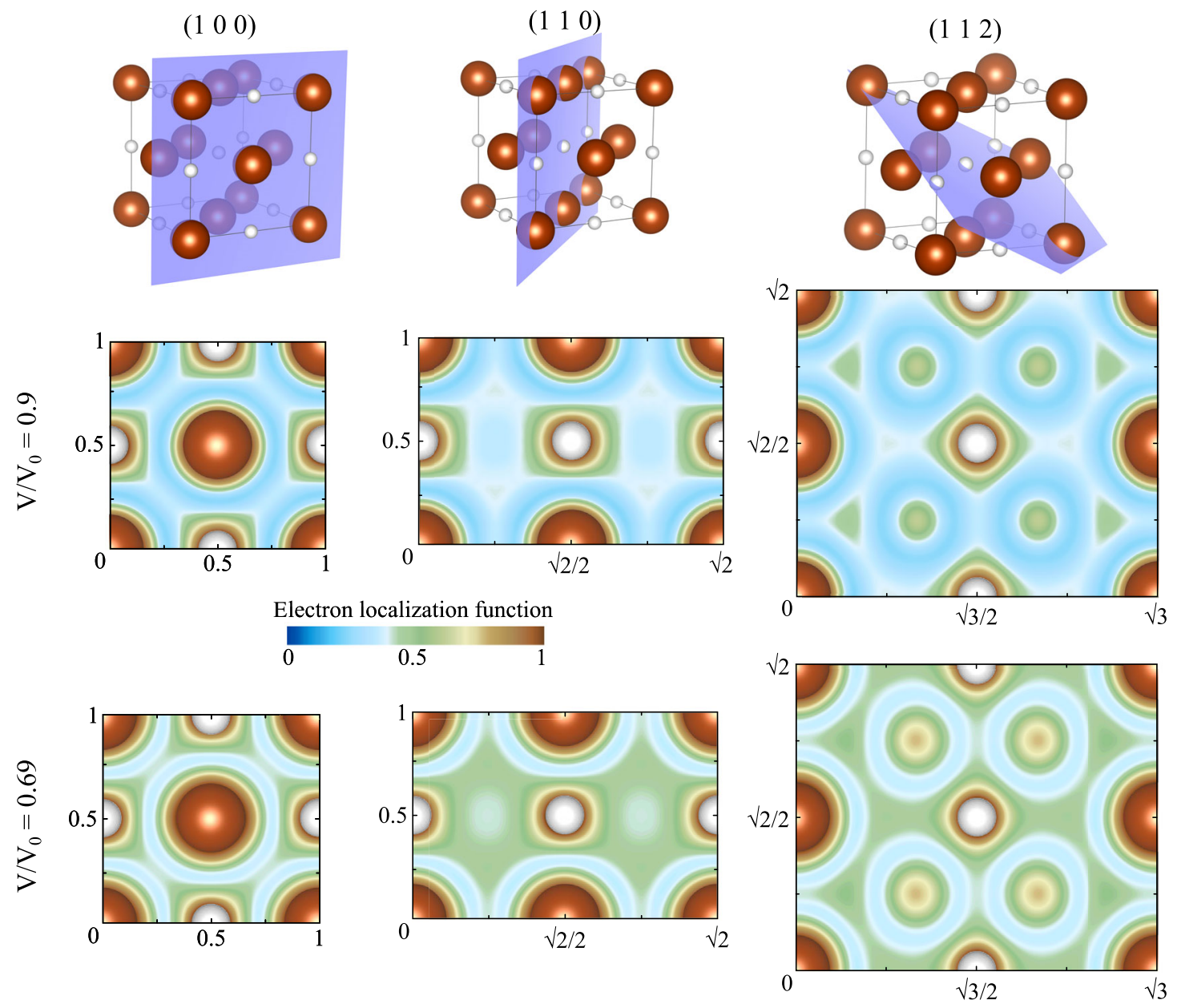

FIG. 5. Electron localization function maps in three major crystal planes at a compression of $V / V_{0}=0.9$ and 0.69 , corresponding to 30 and $200 \mathrm{GPa}$, respectively (for further compression points, see Movie S1 in Supplemental Material [53]). Brown spheres represent iron atoms, white spheres hydrogen. Green areas (ELF $\sim 0.5$ ) correspond to Fermi-gas-like regions of free electrons.

observed for $V / V_{0}=0.88$ coincides with the increase of electron density in the interstitial regions of $\mathrm{FeH}$. The reemergence of Fermi-gas behavior above $110 \mathrm{GPa}$ corresponds to the connection of these areas of enhanced electron density. The pronounced increase in $N_{H-1 s}\left(E_{F}\right)$ also correlates qualitatively with both the formation and connection of these areas.

\section{CONCLUSIONS}

Combining experimental observations from NMR measurements and $a b$ initio computational results, we propose the following qualitative explanation for the high- $P$ evolution of $\mathrm{FeH}$ : With increasing $P$, the electron density from partially uncompensated $3 d-t_{2 g} \mathrm{Fe}$ orbitals in $\mathrm{FeH}$ is pushed towards the hydrogen electron clouds. The successive addition of charges leads to the formation of an intercalating free-electron sublattice of connected hydrogen atoms, exhibiting a continuous enhancement of the electronic density of states of hydrogen at $E_{F}$. Our data suggest that this effect can be observed by NMR when average $\mathrm{H}-\mathrm{H}$ distances become smaller than approximately $2.5 \AA$, which is significantly larger than expected based on previous computational work.

This result suggests that $P$-induced interactions between hydrogen and the transition metal atoms as well as between $\mathrm{H}$ atoms themselves can occur and influence electronic properties of metal hydrides at much lower $P$ than previously anticipated. The experimental findings, supported by $a b$ initio calculations, suggest that other candidates for phonon-mediated high- $T_{c}$ superconductivity may be found far off the proclaimed "lability belt," including the rare-earth sodalite-clathrate superhydrides [54].

The data supporting the findings of this study are available from the corresponding author upon reasonable request.

\section{ACKNOWLEDGMENTS}

We thank Nobuyoshi Miyajima and Katharina Marquardt for help with the FIB milling. We also thank Caterina Melai, Serena Dominijanni, and Catherine McCammon for help 
with XRD measurements. N. D. and L. D. thank the German Research Foundation [Deutsche Forschungsgemeinschaft (DFG) Projects No. DU 954/11-1, No. DU 393/13-1, and No. DU 393/9-2] and the Federal Ministry of Education and Research, Germany (BMBF, Grant No. 5K16WC1) for financial support. S. P. and F. T. were financed by the German Research Foundation (DFG Projects No. PE 2334/1-1 and No. STE 1105/13-1, both in the research unit FOR 2440).

T. M. and L. D. designed the experiment. T. M., S. K., and S.P. prepared the DACs and NMR resonators. S. K. performed focused ion beam cutting as well as structure refinement after laser heating. L. D. performed the sample synthesis in the DACs. S. C. and T. F. as well as S. D. and C. M. performed XRD measurements at DESY and ESRF, respectively. F. T. and G. S.-N. performed the calculations and analyzed the results. T. M., F. T., G. S.-N., N. D. and L. D. performed the data analysis and wrote the manuscript.

[1] R. Mohtadi and S. I. Orimo, The Renaissance of Hydrides as Energy Materials, Nat. Rev. Mater. 2, 16091 (2016).

[2] L. Zhang, Y. Wang, J. Lv, and Y. Ma, Materials Discovery at High Pressures, Nat. Rev. Mater. 2, 17005 (2017).

[3] L.P. Gor'kov and V.Z. Kresin, Pressure and High-T Superconductivity in Sulfur Hydrides, Sci. Rep. 6, 25608 (2016).

[4] E. Zurek, R. Hoffmann, N. W. Ashcroft, A. R. Oganov, and A. O. Lyakhov, A Little Bit of Lithium Does a Lot for Hydrogen, Proc. Natl. Acad. Sci. U.S.A. 106, 17640 (2009).

[5] T. Jarlborg and A. Bianconi, Breakdown of the Migdal Approximation at Lifshitz Transitions with Giant Zero-Point Motion in the $\mathrm{H}_{3} \mathrm{~S}$ Superconductor, Sci. Rep. 6, 24816 (2016).

[6] A. Bianconi and T. Jarlborg, Superconductivity above the Lowest Earth Temperature in Pressurized Sulfur Hydride, Europhys. Lett. 112, 37001 (2015).

[7] A. P. Drozdov, M. I. Eremets, and I. A. Troyan, Superconductivity above $100 \mathrm{~K}$ in $\mathrm{PH}_{3}$ at High Pressures., arXiv:1508.06224.

[8] M. Somayazulu, M. Ahart, A. K. Mishra, Z. M. Geballe, M. Baldini, Y. Meng, V. V. Struzhkin, and R. J. Hemley, Evidence for Superconductivity above $260 \mathrm{~K}$ in Lanthanum Superhydride at Megabar Pressures, Phys. Rev. Lett. 122, 027001 (2019).

[9] A. P. Drozdov, M. I. Eremets, I. A. Troyan, V. Ksenofontov, and S. I. Shylin, Conventional Superconductivity at 203 Kelvin at High Pressures in the Sulfur Hydride System, Nature (London) 525, 73 (2015).

[10] D. V. Semenok, I. A. Kruglov, A. G. Kvashnin, and A. R. Oganov, On Distribution of Superconductivity in Metal Hydrides, arXiv:1806.00865.

[11] D. V. Semenok, A. G. Kvashnin, I. A. Kruglov, and A. R. Oganov, Actinium Hydrides $\mathrm{AcH}_{10}, \mathrm{AcH}_{12}$, and $\mathrm{AcH}_{16}$ as High Temperature Conventional Superconductors, J. Phys. Chem. Lett. 9, 1920 (2018).
[12] T. Meier, N. Wang, D. Mager, J. G. Korvink, S. Petitgirard, and L. Dubrovinsky, Magnetic Flux Tailoring through Lenz. Lenses for Ultrasmall Samples: A New Pathway to HighPressure Nuclear Magnetic Resonance, Sci. Adv. 3, eaao5242 (2017).

[13] T. Meier, S. Petitgirard, S. Khandarkhaeva, and L. Dubrovinsky, Observation of Nuclear Quantum Effects and Hydrogen Bond Symmetrisation in High Pressure Ice, Nat. Commun. 9, 2766 (2018).

[14] T. Meier, S. Khandarkhaeva, S. Petitgirard, T. Körber, A. Lauerer, E. Rössler, and L. Dubrovinsky, NMR at Pressures up to 90 GPa, J. Magn. Reson. 292, 44 (2018).

[15] G. C. Carter, L. H. Bennett, and D. J. Kahan, Metallic Shifts in NMR, first ed. (Pergamon Press, Oxford, 1977).

[16] S. H. Baek, H. Lee, S. E. Brown, N. J. Curro, E. D. Bauer, F. Ronning, T. Park, and J. D. Thompson, NMR Investigation of Superconductivity and Antiferromagnetism in $\mathrm{CaFe}_{2} \mathrm{As}_{2}$ under Pressure, Phys. Rev. Lett. 102, 227601 (2009).

[17] N. Fujiwara, N. Mori, Y. Uwatoko, T. Matsumoto, N. Motoyama, and S. Uchida, Spin Fluctuation in $\mathrm{Sr}_{2} \mathrm{Ca}_{12} \mathrm{Cu}_{24} \mathrm{O}_{41}$ under High Pressure up to $3.0 \mathrm{GPa}$, Physica (Amsterdam) 388C-389C, 229 (2003).

[18] T. Meier, Journey to the Centre of the Earth: Jules Vernes' Dream in the Laboratory from an NMR Perspective, Prog. Nucl. Magn. Reson. Spectrosc. 106-107, 26 (2018).

[19] T. Meier, S. Reichardt, and J. Haase, High-Sensitivity NMR beyond 200,000 Atmospheres of Pressure, J. Magn. Reson. 257, 39 (2015).

[20] F. Peng, Y. Sun, C. J. Pickard, R. J. Needs, Q. Wu, and Y. Ma, Hydrogen Clathrate Structures in Rare Earth Hydrides at High Pressures: Possible Route to Room-Temperature Superconductivity, Phys. Rev. Lett. 119, 107001 (2017).

[21] O. Narygina, L. S. Dubrovinsky, C. A. McCammon, A. Kurnosov, I. Y. Kantor, V. B. Prakapenka, and N. A. Dubrovinskaia, X-Ray Diffraction and Mössbauer Spectroscopy Study of fcc Iron Hydride FeH at High Pressures and Implications for the Composition of the Earth's Core, Earth Planet. Sci. Lett. 307, 409 (2011).

[22] C. M. Pépin, A. Dewaele, G. Geneste, P. Loubeyre, and M. Mezouar, New Iron Hydrides under High Pressure, Phys. Rev. Lett. 113, 265504 (2014).

[23] I. Kantor, V. Prakapenka, A. Kantor, P. Dera, A. Kurnosov, S. Sinogeikin, N. Dubrovinskaia, and L. Dubrovinsky, BX90: A New Diamond Anvil Cell Design for X-Ray Diffraction and Optical Measurements, Rev. Sci. Instrum. 83, 125102 (2012).

[24] Y. Akahama and H. Kawamura, High-Pressure Raman Spectroscopy of Diamond Anvils to 250 GPa: Method for Pressure Determination in the Multimegabar Pressure Range, J. Appl. Phys. 96, 3748 (2004).

[25] Y. Akahama and H. Kawamura, Pressure Calibration of Diamond Anvil Raman Gauge to 310 GPa, J. Appl. Phys. 100, 043516 (2006).

[26] M. S. Conradi, M. P. Mendenhall, T. M. Ivancic, E. A. Carl, C. D. Browning, P. H. L. Notten, W. P. Kalisvaart, P. C. M. M. Magusin, R. C. Bowman, S. J. Hwang, and N. L. Adolphi, NMR to Determine Rates of Motion and Structures in Metal-Hydrides, J. Alloys Compd. 446-447, 499 (2007).

[27] G. Maniara, K. Rajamoorthi, S. Rajan, and G. W. Stockton, Method Performance and Validation for Quantitative 
Analysis by ${ }^{1} \mathrm{H}$ and ${ }^{3} 1 \mathrm{P}$ NMR Spectroscopy. Applications to Analytical Standards and Agricultural Chemicals, Anal. Chem. 70, 4921 (1998).

[28] E. C. Thompson, A. H. Davis, W. Bi, J. Zhao, E. E. Alp, D. Zhang, E. Greenberg, V. B. Prakapenka, and A. J. Campbell, High-Pressure Geophysical Properties of Fcc Phase $\mathrm{FeH}_{X}$, Geochem. Geophys. 19, 305 (2018).

[29] T. Komabayashi and Y. Fei, Internally Consistent Thermodynamic Database for Iron to the Earth's Core Conditions, J. Geophys. Res. Solid Earth 115, B03202 (2010).

[30] P. Giannozzi et al., QUANTUM ESPRESSO: A Modular and Open-Source Software Project for Quantum Simulations of Materials, J. Phys. Condens. Matter 21, 395502 (2009).

[31] P. Giannozzi et al., Advanced Capabilities for Materials Modelling with Quantum ESPRESSO, J. Phys. Condens. Matter 29, 465901 (2017).

[32] P. E. Blöchl, Projector Augmented-Wave Method, Phys. Rev. B 50, 17953 (1994).

[33] J. P. Perdew, K. Burke, and M. Ernzerhof, Generalized Gradient Approximation Made Simple, Phys. Rev. Lett. 77, 3865 (1996).

[34] L. Stixrude, Structure of Iron to 1 Gbar and 40000 K, Phys. Rev. Lett. 108, 055505 (2012).

[35] H. J. Monkhorst and J. D. Pack, Special Points for BrillouinZone Integrations, Phys. Rev. B 13, 5188 (1976).

[36] A. D. Becke and K. E. Edgecombe, A Simple Measure of Electron Localization in Atomic and Molecular Systems, J. Chem. Phys. 92, 5397 (1990).

[37] C. Elsässer, J. Zhu, S. G. Louie, B. Meyer, M. Fähnle, and C. T. Chan, Ab Initio Study of Iron and Iron Hydride: II. Structural and Magnetic Properties of Close-Packed Fe and FeH, J. Phys. Condens. Matter 10, 5113 (1998).

[38] C. Elsässer, H. Krimmel, M. Fähnle, S. G. Louie, and C. T. Chan, Ab Initio Study of Iron and Iron Hydride: III. Vibrational States of $\mathrm{H}$ isotopes in $\mathrm{Fe}, \mathrm{Cr}$ and $\mathrm{Ni}$, J. Phys. Condens. Matter 10, 5131 (1998).

[39] W. D. Knight, Nuclear Magnetic Resonance Shift in Metals, Phys. Rev. 76, 1259 (1949).

[40] C. H. Townes, C. Herring, and W. D. Knight, The Effect of Electronic Paramagnetism on Nuclear Magnetic Resonance Frequencies in Metals, Phys. Rev. 77, 852 (1950).
[41] R. Goring, R. Lukas, and K. Bohmhammel, Multipulse NMR Investigation of Band Structure in Titanium Hydride: Proton Knight Shift and Spin-Lattice Relaxation, J. Phys. C 14, 5675 (1981).

[42] S. Kazama and Y. Fukai, Local Electronic Environment of Protons in $\mathrm{VH}_{x}$ Alloys: Knight Shift and $\mathrm{T}_{1}$ of Proton NMR, J. Phys. Soc. Jpn. 42, 119 (1977).

[43] S. Kazama and Y. Fukai, High Resolution Measurements of Proton NMR in $\mathrm{VH}_{x}, \mathrm{NbH}_{x}$ and $\mathrm{TaH}_{x}$ : Proton Knight Shift and Magnetic Susceptibility, J. Less-Common Met. 53, 25 (1977).

[44] D. S. Schreiber and L. D. Graham, On the Proton Knight Shift in Metallic $\mathrm{VH}_{0.66}$, J. Chem. Phys. 43, 2573 (1965).

[45] J. Korringa, Nuclear Magnetic Relaxation and Resonance Line Shift in Metals, Physica (Utrecht) 16, 601 (1950).

[46] G. Czycholl, Theoretische Festkörperphysik, third ed. (Springer, Berlin, 2008).

[47] Y. Yafet and V. Jaccarino, Nuclear Spin Relaxation in Transition Metals, Phys. Rev. 133, A1630 (1964).

[48] A. Narath and H. T. Weaver, Effects of Electron-Electron Interactions on Nuclear Spin-Lattice Relaxation Rates and Knight Shifts in Alkali and Noble Metals, Phys. Rev. 175, 373 (1968).

[49] W. Heitler and E. Teller, Time Effects in the Magnetic Cooling Method. I, Proc. R. Soc. A 155, 629 (1936).

[50] S. D. Goren, C. Korn, H. Riesemeier, E. Rossler, and K. Lüders, Titanium Knight Shift in Titanium Hydride, Phys. Rev. B 34, 6917 (1986).

[51] G. E. Volovik, Topological Lifshitz Transitions, Low Temp. Phys. 43, 47 (2017).

[52] I. M. Lifshitz, Anomalies of Electron Characteristics of a Metal in the High Pressure Region, Sov. Phys. JETP 11, 1130 (1960).

[53] See Supplemental Material at http://link.aps.org/ supplemental/10.1103/PhysRevX.9.031008 for animation of all ELF maps and electronic density of states calculations in combination with experimental data.

[54] C. Heil, S. Di Cataldo, G. B. Bachelet, and L. Boeri, Superconductivity in Sodalite-like Yttrium Hydride Clathrates, Phys. Rev. B 99, 220502 (2019). 